\title{
Parting smoothly?
}

Nicholas SHackel

In 'How to part ways smoothly' Hud Hudson (2007) presents 'two temporally-continuous spatially unextended material objects that ... share all of their temporal parts up until their very last time-slice' (2007: 156). They share their location throughout all but the last instant of their lives, at which instant they are a metre apart. Hudson claims that they part smoothly. I shall show that they don't.

The two objects are named Three and Nine. Three is the diachronic fusion of the objects named Common and Here, Nine is the diachronic fusion of Common and There. 
Common is ... always on the move. At $t_{0}$ (the first moment of his existence), Common sits at the 12:00 point on a one metre diameter wall clock. He Zeno-sprints around the perimeter of the clock from $t_{0}$ up to but not including $t_{100}$ (the first moment of his non-existence). That is, during the first $1 / 2$ of his life he moves from the 12:00 point to the 3:00 point, during the next 1/4 of his life he moves from 3:00 to 6:00, during the next 1/8 of his life he moves from 6:00 to 9:00, during the next 1/16 he moves from 9:00 to 12:00...and so on.... he happily passes every point on the perimeter infinitely many times. (2007: 156)

At $t_{100}$, however, there are two instantaneous, point-sized objects, one positioned at the 3:00 point and one positioned at the 9:00 point on our wall clock. Name them Here and There. (2007: 157)

By parting smoothly Hudson means that they part whilst 'neither ... engages in discontinuous motion' (2007: 156). ${ }^{1}$ For an object's motion to be continuous is for there to be a continuous function from the times of its existence to its spatial position. So consider the function, $f$, that maps the times of Three's existence, the interval $\left[t_{0}, t_{100}\right]$, to its spatial position on the clock face. If Three is temporally continuous then $f$ is a continuous function, and so is continuous at $t_{100 .}{ }^{2} f$ is continuous at $t_{100}$ iff

for any $\varepsilon>0$ there exists a $\delta>0$ such that for all times, $t \in\left[t_{0}, t_{100}\right]$, if $\left|t_{100}-t\right|<\delta$ then $\left|f\left(t_{100}\right)-f(t)\right|<\varepsilon .^{3}$

I shall now show that the later proposition is false, that is to say, I shall show that

(1) there is an $\varepsilon>0$ such that for all $\delta>0$ there exists a time, $t \in\left[t_{0}, t_{100}\right]$, such that $\left|t_{100}-t\right|<\delta$ and $\left|f\left(t_{100}\right)-f(t)\right|>\varepsilon$

Hence I will have shown that Three moves discontinuously, and a similar proof shows the same for Nine.

${ }^{1}$ Mathematicians use 'smooth' to mean more than continuous: a smooth function has continuous derivatives up to the $n$th derivative for some $n$ or for all $n$ (see, e.g. http://mathworld.wolfram.com/SmoothFunction.html). In this sense, the continuity of a function does not imply its smoothness, whereas Hudson's argument requires continuity of motion to imply smoothness of motion. So I do not think we should interpret him in those terms, and in fact I think it is clear that Hudson is using 'smooth' colloquially to mean, and to mean no more than, continuous.

${ }^{2}$ A function is continuous iff it is continuous at every point in its domain.

${ }^{3}$ See, e.g., the general definition of continuity at a point for mappings of metric spaces, Kolmogorov and Fomin 1975: 44 (\$5.2). 
I prove this technically below, but the quick way to see the result is this. For $f$ to be continuous at $t_{100}$ means that as the time approaches $t_{100}$, Three gets and remains arbitrarily close to its position at $t_{100}$. Getting and remaining arbitrarily close means that for any distance from its position at $t_{100}$, however small, there is a correlate (possibly very small) interval of time ending at $t_{100}$, during which interval Three is always within that distance of its position at $t_{100}$. Now if we consider the way that Three is at the point 9:00 on the clock face infinitely many times before $t_{100}$, it is evident that in any interval of time leading up to $t_{100}$, however small we make it, there is a time at which Three is at 9:00 on the clock face, which is to say, one metre from its final position, and so Three does not remain arbitrarily close to its final position.

So Hudson is mistaken in his characterization of continuous motion when he says

neither character ever moves discontinuously, for no matter how small the open region you take about the space-time point occupied by Here you find a temporal slice of Three present in that region prior to $t_{100}$. (2007: 157$)$

This satisfies a necessary condition for continuous motion, namely, getting arbitrarily close, but fails the condition of remaining arbitrarily close. If this were sufficient for continuous motion, a particle whose position on the $x$-axis was given by the function

$$
\begin{aligned}
& p:[0,1] \rightarrow \mathbb{R} \\
& p(t)=\left\{\begin{array}{l}
1-t \text { for } t \in[0,1] \backslash\left\{t: t=1 / 2^{n} \text { for } n \in \mathbb{N}\right\} \\
1 / t \text { for } t \in\left\{t: t=1 / 2^{n} \text { for } n \in \mathbb{N}\right\}
\end{array}\right.
\end{aligned}
$$

would be moving continuously while approaching zero, when in fact its motion is radically discontinuous (as it approaches zero there are instants at which it is arbitrarily far away, since for any $x \in \mathbb{R}$ there is an $n \in \mathbb{N}$ such that $2^{n}>x$ ). Clearly similar cases could be formulated in which intervals during which the particle approaches zero alternate with intervals during which the particle occupies positions arbitrarily far away. Consequently the problem could not be avoided by redefining continuous motion in terms of continuous motion almost everytime (i.e. being allowed to exclude sets of times of measure zero).

Proof of (1): Let $\left\{t_{k}\right\}$ be the sequence of times at which Three is at 9:00 on the clock face. This sequence is defined by

$$
t_{k}=t_{100}\left(1-\frac{1}{2^{4 k-1}}\right)
$$


Since for all $k$ in $\mathbb{N}, f\left(t_{k}\right)=9: 00$, and $f\left(t_{100}\right)=3: 00$, we have for all $k$ in $\mathbb{N}$ $\left|f\left(t_{100}\right)-f\left(t_{k}\right)\right|=1>1 / 2$. So setting $\varepsilon=1 / 2$ and $t=t_{j},\left(t_{j} \in\left\{t_{k}\right\}\right)$, to prove (1) we now need only to show that

(2) for all $\delta>0$ there exists a $j \in \mathbb{N}$ such that $\left|t_{100}-t_{j}\right|<\delta$.

(2) will follow if $t_{100}$ is the limit of the sequence $\left\{t_{k}\right\}$, which it is: 4

$$
\begin{aligned}
\lim _{\mathrm{k} \rightarrow \infty} t_{k} & =\lim _{\mathrm{k} \rightarrow \infty}\left(t_{100}\left(1-\frac{1}{2^{4 k-1}}\right)\right) \\
& =t_{100} \lim _{\mathrm{k} \rightarrow \infty}\left(1-\frac{1}{2^{4 k-1}}\right) \\
& =t_{100}
\end{aligned}
$$

By definition, $t_{100}$ is the limit of the sequence $\left\{t_{k}\right\}$ iff for all $\delta>0$ there exists an $m \in \mathbb{N}$ such that for all $k>m$, $\left|t_{100}-t_{k}\right|<\delta$

So given any $\delta$ we simply choose any $j$ greater than the corresponding $m \in \mathbb{N}$ and for that $j,\left|t_{100}-t_{j}\right|<\delta$, whence we have (2).

FHI, Faculty of Philosophy, University of Oxford
16 St Ebbes St, Oxford OX1 1PT, UK
nicholas.shackel@philosophy.ox.ac.uk

\section{References}

Binmore, K. G. 1982. Mathematical Analysis. Cambridge: Cambridge University Press. Hudson, H. 2007. How to part ways smoothly. Analysis 67: 156-57.

Kolmogorov, A. N. and S. V. Fomin. 1975. Introductory Real Analysis. Tr. R. A. Silverman. New York: Dover.

${ }^{4}$ First step by the combination theorem (Binmore 1982: $\left.30 \$ 4.8\right)$. Second step by the sandwich theorem (Binmore 1982: $31 \$ 4.10$ ): for all $k, 1-\frac{1}{2^{k}} \leq 1-\frac{1}{2^{4 k-1}} \leq 1$, and both the sequences $\left\{1-\frac{1}{2^{k}}\right\}$ and $\{1\}$ converge to 1 . 